\title{
Un apunte sobre Spinoza y la tolerancia: a propósito de una interpretación reciente
}

\author{
A note on Spinoza and tolerance: \\ about a recent interpretation \\ Uma observação sobre Spinoza e tolerância: \\ sobre uma interpretação recente
}

LUIS PLACENCIA

\section{Resumen}

En este texto quisiera desmontar la lectura de Spinoza sugerida por M. Svensson en su recientemente premiado ensayo "Una disposición pasajera". En el mencionado texto Svensson vincula la posición sobre la tolerancia que habría tenido Spinoza con aquella que J. Locke desarrollara en su conocida carta sobre el particular. Ambas corresponderían a posiciones que habrían defendido un cierto "minimalismo doctrinal" destinado a evitar conflictos. Svensson opone esta concepción a las visiones de autores contemporáneos como Derrida que tenderían a intentar situarse "más allá de la tolerancia", por un lado, así como a una concepción robusta de la tolerancia que él identifica con Agustín, siendo esta última, a sus ojos, la mejor. Svensson respalda su lectura de Spinoza principalmente en una interpretación del capítulo XIV del Tratado teológico político que presenta los siete dogmas de la fe universal (fidei universalis dogmata) expuestos ahí como una suerte de teología mínima que impediría los conflictos religiosos. Lo anterior sería coincidente con una concepción de la tolerancia como virtud que busca ante todo la desaparición del conflicto vía la eliminación de concepciones teológicas sustantivas (esto se puede ampliar a una concepción de la tolerancia ya no meramente en el ámbito de la teología, sino que también como una suerte de virtud moral consistente en aceptar la neutralidad moral de

a Universidad de Chile, Santiago de Chile, Chile. Doctor en Filosofía, e-mail: luis.placencia@uchile.cl 
cualquier visión que no transgreda ciertos mínimos). En este trabajo busco mostrar algunos malentendidos que están, según me parece, a la base de la lectura de Svensson y junto con ello ofrecer una mejor interpretación.

Palabras clave: Tolerancia. Spinoza. Tratado teológico-político.

\begin{abstract}
In this text I would like to dismantle Spinoza's reading suggested by M. Svensson in his recently awarded essay "Una disposición pasajera". In the aforementioned text Svensson links the position on tolerance that Spinoza would have had with that which J. Locke developed in his well-known letter on the subject. Both would correspond to positions that would have defended a certain "doctrinal minimalism" aimed at avoiding conflicts. Svensson opposes this conception to the visions of contemporary authors such as Derrida who would tend to try to place themselves "beyond tolerance", on the one hand, as well as to a robust conception of tolerance that he identifies with Augustine, the latter being, in his eyes, the best. Svensson supports his reading of Spinoza mainly in an interpretation of chapter XIV of the Political Theological Treaty which presents the seven dogmas of the universal faith (fidei universalis dogmata) exposed there as a sort of minimal theology that would prevent religious conflicts. This would coincide with a conception of tolerance as a virtue that seeks above all the disappearance of conflict via the elimination of substantive theological conceptions (this can be extended to a conception of tolerance no longer merely in the realm of theology, but also as a sort of moral virtue consisting in accepting the moral neutrality of any vision that does not transgress certain minimums). In this paper I seek to show some misunderstandings that are at the basis of Svensson's reading and along with it offer a better interpretation.
\end{abstract}

Keywords: Tolerance. Spinoza. Political Theological Treatise.

\title{
Resumo
}

Neste texto, gostaria de desmontar a leitura de Spinoza sugerida por M. Svensson em seu recém-premiado ensaio "Una disposición pasajera". No texto acima mencionado, Svensson vincula a posição sobre a tolerância que Espinoza teria tido com a que J. Locke desenvolveu na sua conhecida carta sobre o assunto. Ambos corresponderiam a posições que teriam defendido um certo "minimalismo doutrinário" destinado a evitar conflitos. Svensson opõe esta concepção às visões de autores contemporâneos como Derrida, que tenderiam a tentar colocar-se "além da tolerância", por um lado, e a uma concepção robusta de tolerância que ele identifica com Agostinho, sendo este último, aos seus olhos, o melhor. Svensson apoia sua leitura de Espinoza principalmente em uma interpretação do capítulo XIV do Tratado Teológico Político que apresenta os sete dogmas da fé universal (fidei universalis dogmata) ali expostos como uma espécie de teologia mínima que evitaria conflitos religiosos. Isto coincidiria com uma concepção de tolerância como uma virtude que procura sobretudo o desaparecimento do conflito 
através da eliminação das concepções teológicas substantivas (isto pode ser alargado a uma concepção de tolerância já não só no domínio da teologia, mas também como uma espécie de virtude moral que consiste em aceitar a neutralidade moral de qualquer visão que não transgrida certos mínimos). Neste documento, procuro mostrar alguns mal-entendidos que, a meu ver, estão na base da leitura de Svensson e, juntamente com ela, oferecem uma melhor interpretação.

Palavras-chave: Tolerância. Spinoza. Tratado Teológico Político.

\section{Una discusión reciente del concepto de tolerancia}

El concepto de "tolerancia" suele ser invocado en las discusiones públicas. Al hacerlo, quienes lo emplean, suelen hacer como si su significado fuera completamente transparente. Sin embargo, como suele ocurrir en el caso de los conceptos interesantes para la filosofía, el asunto es más bien al revés: el concepto no es del todo claro y un análisis somero de lo que normalmente se ha dicho de él en la tradición de discusión sobre esta noción lo deja bastante claro. Es por ello que la filosofía ha dedicado, desde hace bastante tiempo, no pocos esfuerzos a elucidar el significado de este término.

En un ensayo relativamente reciente, publicado en 2013 y premiado con anterioridad en la versión 2011 del concurso de Ensayo convocado por la Universidad Diego Portales, el Goethe Institut y el suplemento “Artes y Letras" del diario El Mercurio, el profesor chileno Manfred Svensson ha intentado defender una concepción de la tolerancia que ha opuesto a, por un lado, las posiciones que pretenden situarse, de acuerdo a una clasificación que él mismo intenta en su texto, "más allá de la tolerancia" (como por ejemplo sería el caso de la de Derrida) y a aquellas que corresponderían a posiciones situadas "más acá” de ella (posiciones que identifica con una "tradición liberal erasmiana" a la que pertenecerían autores como Hobbes, Locke y Spinoza). En este texto quisiera desmontar la lectura de Spinoza sugerida por Svensson para luego referirme a algunos malentendidos que están a la base de la misma.

Para lograr este objetivo debo, en primera instancia, referirme brevemente a la posición de Svensson. Su punto de partida es la idea de que la tolerancia es una 
virtud, aunque no sea claro ni 1) qué tipo de virtud sea ni 2) hasta qué punto debe ella ser ejercida. El mismo Svensson es consciente de que no es una visión compartida por todos los autores el hecho de que la tolerancia sea una virtud (como excepción, por ejemplo, nombra él mismo a Nietzsche), pero indica de entrada que él se sitúa en la tradición opuesta de comprensión de este concepto. En el marco de esta tradición él buscará dar una respuesta a la primera de las cuestiones mencionadas, i.e. qué tipo de virtud es la tolerancia, dejando de lado la segunda. Lo anterior parece justificarse, a los ojos de Svensson, en el hecho de que en el debate actual, especialmente en el ámbito de la discusión pública, la cuestión que se debate de modo prácticamente único es más bien la segunda, sc. la cuestión del alcance de la tolerancia, mientras que pareciera que en ese debate se dejaría intacta la cuestión más básica de qué sería aquello sobre cuyo alcance se discute (Svensson 2013, 2324). ${ }^{1}$ Con el objetivo de responder a la pregunta por el tipo de virtud que sería la tolerancia, Svensson analiza aquí tres clases de tradiciones respecto de la tolerancia que se habrían documentado a lo largo de la historia de la filosofía. La primera de ellas es sin duda la más conocida y también la que me interesa aquí: se trata de aquella que es representada, según Svensson, por Locke fundamentalmente en su conocidísima Carta sobre la tolerancia, pero también, según él, por Spinoza en su Tratado teológico-politico (SVENSSON, 2011, p. 130; SVENSSON, 2013, p. 28). Sobre ella indica Svensson "la posición que vemos ahí presenta ciertos rasgos que la hacen parecer menos que tolerancia, en cuanto dichos rasgos tienden a la simple reducción de los conflictos, reduciendo así las instancia en que la tolerancia debe ser practicada" (SVENSSON, 2013, p. 26). Svensson, de hecho ve en esta concepción una posición errada que en último término tiene como consecuencia la confusión de la tolerancia "con actitudes como la apertura o el respeto" (SVENSSON, 2013, p. 32). La caracterización más precisa de esta posición transcurre apelando fundamentalmente a la Carta de Locke, aunque Svensson presenta explícitamente parte de los resultados de su análisis de la Carta como tesis que compartiría Spinoza, especialmente en lo que concierne a la tercera nota que halla en la caracterización

\footnotetext{
1 Me referiré aquí fundamentalmente al ya mencionado trabajo de Svensson, aunque también a Svensson (2011) donde desarrolla parte de las ideas contenidas en Svensson (2013).
} 
lockeana de la tolerancia, tal como ella se revela en el examen que Svensson hace de ella. En esta caracterización más precisa Svensson destaca, en primera instancia, como característica definitoria del concepto de tolerancia los siguientes aspectos:

a) La tolerancia es elevada al plano de ser la virtud fundamental, movimiento en el que ve una ruptura con la tradición precedente (SVENSSON, 2013, p. 30).

Tras este movimiento Svensson ve el inicio de una tendencia que desembocará en la idea de la tolerancia como una virtud independiente, pero que se manifiesta en su fase inicial en Locke

b) Como una fusión de la tolerancia con las demás virtudes fundamentales (SVENSSON, 2013, p. 30).

De acuerdo con Svensson, lo anterior está vinculado con una tercera nota, sc.

c) El minimalismo doctrinal (SVENSSON, 2013, p. 33).

Este punto estaría directamente vinculado con el objetivo central de la Carta que sería la defensa de la tolerancia religiosa, sentido del término que, como se sabe, es justamente el más común en la época. De esta manera, la defensa del "minimalismo doctrinal" se halla directamente vinculada - para no decir exclusivamente - con la defensa de un minimalismo teológico. Tal minimalismo doctrinal estaría, entonces, directamente vinculado con la defensa de una "ortodoxia mínima" (SVENSSON, 2013, p. 34), ortodoxia que haría posible resolver el problema central que enfrentan los autores que dedicaron pensamientos al problema de la tolerancia en el S. XVII, sc. dar cuenta de la posibilidad de la convivencia de más de un credo religioso en una misma comunidad política. Es justamente en este punto donde Svensson ve un punto de contacto entre Locke y sus contemporáneos, en concreto con Spinoza. Así indica Svensson lo siguiente:

En el continente, en tanto, Spinoza argumentaba en términos similares en su Tratado teológico-político: para fundamentar la tolerancia reduce todo el campo doctrinal a la existencia de un Dios único, bueno, al cual hay que agradar con justicia y caridad. La finalidad de tal reducción doctrinal es, en palabras del mismo Spinoza, porque así 'no queda lugar alguno para las controversias' (SVENSSON, 2013, p. 35).

Svensson respalda su lectura, como parece ser claro, en una interpretación del capítulo XIV del TTP que interpreta los siete dogmas de la fe universal fidei universalis dogmata) expuestos ahí como una suerte de teología mínima que impediría 
los conflictos religiosos. Esto sería coincidente con una concepción de la tolerancia como virtud que busca ante todo la desaparición del conflicto vía la eliminación de concepciones teológicas sustantivas (esto se puede ampliar a una concepción de la tolerancia ya no meramente en el ámbito de la teología, sino que también como una suerte de virtud moral consistente en aceptar la neutralidad moral de cualquier visión que no transgreda ciertos mínimos).

Contra esta interpretación de la tolerancia Svensson objeta varios puntos. En su opinión ella parece suponer que la controversia no puede ser provechosa y que los conflictos religiosos o morales no tienen solución, de suerte que sólo se podría apelar a su cancelación vía el establecimiento de mínimos. Esto supone, en rigor, piensa Svensson, y en esto creo que tiene razón, ni siquiera alcanzar el nivel de la tolerancia, que parece ser justamente la capacidad de aceptar un mal, pues se asume desde la perspectiva de la concepción robusta de la tolerancia que la supresión de lo que considera "malo" o las condiciones de la supresión de ese mal son en algún sentido peores que la existencia de ese mal.

Además de esta visión de la tolerancia, piensa Svensson, se han presentado a lo largo de la historia de la filosofía al menos dos variantes. Una, que en cierta medida es consecuencia de la primera, a los ojos de Svensson, que sería el intento de dejar atrás la tolerancia y superarla por una perspectiva en el trato con lo que no es considerado bueno ni deseable que no implique asumir que se lo debe meramente "soportar". Svensson ve el germen de esta perspectiva ya en la ilustración (SVENSSON, 2013, p. 40 y ss.), aunque ella llegaría a su consumación en las obras de autores contemporáneos como Derrida. A esta tradición de comprensión de la tolerancia como algo a superar, opone Svensson en última instancia la concepción agustiniana de la misma, que él suscribe, y que llama "concepción robusta de mera tolerancia". La agustiniana sería una concepción de "mera tolerancia" en la medida en que no se la situaría más allá de sí misma, mientras que sería robusta, en la medida en que ella no sería ajena a una concepción sustantiva del bien ni tampoco a sus propios presupuestos. Así, la tolerancia no habría de ser entendida como la máxima de las virtudes, sino que tendría que situarse como una virtud secundaria, que es enmarcada en un marco más comprensivo de concepción del bien. 


\section{Observaciones críticas a la interpretación de Svensson}

La interpretación que realiza Svensson de la posición de Spinoza en relación a la tolerancia me parece cuestionable desde muchos puntos de vista. Varios de ellos deberían parecer obvios a quien tenga en vistas la investigación especializada en la

filosofía política de Spinoza en las últimas décadas. ${ }^{2}$ Mencionaré dos de ellos, que tomo de esa discusión, para luego plantear una tercera razón que creo hace de la interpretación Svensson una forma altamente cuestionable de comprender el punto de Spinoza.

El primero de estos puntos se vincula con el modo en como Svensson parece comprender el texto de los 7 dogmas y la función que éste cumpliría. En efecto, aunque existen autores relevantes en la literatura que conceden que lo que ahí se ofrece es algo así como un "mínimo especulativo" (WALTHER, 2014, p. 143 y ss.), me parece que en general la interpretación correcta es aquella que tiende a devaluar el carácter filosófico de tales dogmas y más bien los nivela al plano de preceptos de naturaleza política (MADANES, 2001, p. 164). Dicho de otro modo, de acuerdo a la interpretación que estimo correcta, no se trata con los 7 dogmas de asegurar una teología mínima, sino que la obediencia, i.e. no buscan esos 7 dogmas asentar una suerte de "mínimo teórico" que suprima la posibilidad del conflicto teológico, sino más bien que se debe aceptar que no se puede ser juez en causa propia (dogma 1), que alguien debe ejercer de árbitro inapelable en el conflicto político o jurídico (dogma 2), que no se puede dudar de la equidad de la justicia de ese "alguien" (dogma 3), que el soberano es libre y que todos los demás están obligados a obedecerle (dogma 4), que la liturgia civil corresponde a obedecer la ley (dogma 5), que es necesario que las leyes están respaldadas por un sistema de premios y castigos (dogma 6), que el soberano puede levantar esos castigos (dogma 6). Todas esta manera de interpretar los 7 dogmas es mucho más coincidente que la lectura de Svensson con el modo en que Spinoza interpreta la Escritura (i.e. como no

\footnotetext{
2 El concepto de "tolerancia" y su papel en la obra de Spinoza ha sido ampliamente discutido en los últimos años. Aparte de los textos que menciono aquí, que se refieren fundamentalmente al TTP, se puede el trabajo de Rosenthal (2001) referido a la ética.
} 
conteniendo mensaje especulativo alguno) a lo largo del T'TP. Madanes, cuya interpretación sigo aquí, agrega con razón:

Si se suprime alguna de estas creencias, se suprime la obediencia. Y efectivamente es así. Pues basta con traducir estos dogmas católicos a un lenguaje político para caer en la cuenta de que se trata del esquema hobbesiano. No hay estado de derecho que pueda subsistir si cada uno es juez en casua propia y si no hay legislador soberano con poder coacción. La teología se distingue de la filosofía, pero se asimila a la política. Nuevamente la Biblia se ha reducido a un manual de instrucción cívica (MADANES, 2001, p. 166).

Una segunda línea obvia de objeción, que puede resultar bastante prometedora a primera vista, se basa en tomar en cuenta la interpretación que hace F. Mignini de la noción de "tolerancia" en la obra de Spinoza. Según esta interpretación, y contrariamente a lo que sostiene la mayoría de los autores, el concepto de tolerancia no juega en la obra de Spinoza ningún papel. Mignini aporta para esta tesis evidencia sumamente interesante que es lamentable que autores como Svensson no tomen en cuenta, de modo que me referiré brevemente al contenido de su posición, mas no para suscribirla ni tratarla más en detalle aquí.

La tesis de Mignini consiste en último término en afirmar que

parece legítimo suponer que en el pensamiento spinoziano la noción moderna de tolerancia - entendida como la concesión a otros para pensar y expresarse en materia religiosa de manera diferente y no compartida con nosotros - no tiene la justificación teórica con la que se justifica en otros contextos y que se encuentra, de modo implícito, como ya históricamente superada (MIGNINI, 2009, p. 8-9).

Esta interpretación de Spinoza como un filósofo que se sitúa, en la terminología de Svensson, "más allá" de la tolerancia, cuenta con importante apoyo textual, tal como lo muestra el mismo Mignini, partiendo aquí del hecho básico de que el término mismo tolerantia no es empleado sino una sola vez en la obra de Spinoza y en un contexto diferente, uso que claramente no parece calzar con lo que Svensson identifica con la concepción spinozista de la tolerancia, entre otras cosas porque aquí claramente el término "tolerancia” es empleado para designar la "soportación” de un mal inflingido arbitrariamete. La revisión que realiza Mignini del marco interpretativo de Spinoza lo lleva a concluir que la "tolerancia" como una forma de "soportación" o de "paciencia" 
implica necesariamente una intolerencia teorética, ésta se configura como soportación de un mal considerado como objetivo y por lo tanto no sin el agregado de una cierta tristeza. Pero esa es la primera y fundamental de las pasiones nocivas. Antes bien, es el símbolo mismo de la imperfección, siendo el pasaje de una mayor a una menor perfección (MIGNINI, 2009, p. 39).

Por cierto, en favor de Svensson y su interpretación se podría decir que Mignini y la suya no vienen sino corroborar el mismo diagnóstico sólo que desde una perspectiva diferente: Spinoza no llega a concebir una genuina defensa de la tolerancia (mas no, en el esquema de Svensson, porque ella implique una consideración inadecuada de las cosas, sino que porque ella supondría el puro el acuerdo, aunque no la aceptación del conflicto).

Más que profundizar en la línea de Mignini, que ha sido criticada por autores como Beltrán $(1994)^{3}$, me gustaría concentrarme en un pasaje de la introducción del TTP, cuya recta interpretación me parece de mucha relevancia para desactivar la interpretación de Svensson o al menos para mostrar sus límites. Propongo considerar el pasaje en detalle:

5.- De lo que acabamos de decir sobre la causa de la superstición, se sigue claramente que todos los hombres son por naturaleza esclavos de la superstición, por mucho que algunos digan que creen que la superstición nace de que los mortales tienen una idea confusa de la divinidad. Se sigue, además, que la superstición debe ser tan variada e inconstante, como todos los engaños (ludibria) del espíritu y los furores de la vehemencia y finalmente que ella no puede mantenerse sino gracias a la esperanza, la el odio, la ira y el engaño, ya que no nace de la razón, sino que de la sola pasión (ex solo affectu), y de la más eficaz de todas. De ahí que cuanto más fácil ocurre que los hombres sean tomados por cualquier género de superstición, tanto más difícil es que permanezcan en una y la misma. Por el contrario, dado que el vulgo permanece siempre igual de miserable no alcanza nunca descanso duradero, por lo que siempre lo nuevo y lo nunca lo ha engañado le place en grado sumo. Esta inconstancia ha sido la causa de múltiples disturbios y de guerras atroces, puesto que nada gobierna de modo más eficaz a la multitud que la superstición, tal como ya ha hecho notar de la mejor manera el mismo Quinto Curcio en el libro 4 capítulo 10 de su obra. De ahí que ocurra que el vulgo sea inducido, bajo la especie de la religión, a a dorar a sus reyes como dioses o por el contrario a execrarlos y detestarlos como peste universal del género humano.

6.- A fin de evitar este mal se ha puesto ingente esmero en adornar la religión verdadera o la falsa por medio de un culto y una pompa que le diera prestigio en todo momento y le asegurara veneración de parte de todos. Esto ha sido logrado perfectamente por los turcos, entre los cuales incluso la disputa es un nefanda, y subyugan el juicio de cada uno con tantos prejuicios, que no dejan en el espíritu lugar alguno a la sana razón, ni para cierta duda ${ }^{4}$

Estos pasajes son esenciales para los fines que en el presente texto se han sugerido, fundamentalmente por dos razones: a) en ellos se comienza a mencionar la

\footnotetext{
3 Hay respuesta de Mignini en MIGNINI, 1994.

${ }^{4}$ Cito aquí y en lo que viene en mi propia traducción.
} 
dimensión política de la superstición: ella es un dispositivo afectivo, basado en pasiones humanas como el miedo, el odio, etc; dispositivo que está anclado en la estructura misma del ser humano, tanto en el plano emocional como en el plano epistémico. Este dispositivo tiene un rendimiento político de enorme relevancia, tal como ya lo habría visto el mismo Quinto Curcio, a quien Spinoza cita. Ahora bien, el modo en que este rendimiento político opera en el detalle no es explicado por Spinoza, salvo por una vaga referencia a la institución del culto y de las ceremonias y de la inducción a la veneración o execración de las autoridades, como si fueran dioses o miasmas. La institución del culto busca otorgar estabilidad a lo que es de suyo lábil, puesto que la superstición, debido a su origen, no lleva consigo el germen de la estabilidad. Por ello la institución de las ceremonias y el culto tiene como fin otorgar eficacia política al dispositivo afectivo que genera la superstición, elevando la superstición, por tanto, a un plano ya no puramente afectivo. Tal elevación tiene lugar mediante el "prejuicio", noción que es introducida por primera vez en este texto y que responde justamente a la estabilización de los resultados de la superstición, estabilización que a su vez permite en empleo político de la misma. El prejuicio es, ante todo, un estado epistémico que consiste en tener una opinión falsa sobre Dios o los dioses, opinión que surge del intento de lidiar con la fortuna y con las inseguridades que ella genera. Esta relevante diferencia entre "superstición" y "prejuico" parece haber pasado por alto a casi todos los intérpretes de Spinoza, con la excepción de R. Schnepf, quien ha llamado la atención sobre esta distinción (SCHNEPF, 2014, p. 40). ${ }^{5}$ Tal diferencia se articula fundamentalmente a partir de la naturaleza afectiva de la superstición, por un lado, y la naturaleza epistémica del prejuicio, por el otro, donde no es precisamente el dispositivo afectivo implicado por la superstición el que librado a sí mismo el que genera las dificultades políticas que Spinoza quiere enfrentar en su texto, sino que muy por el contrario, es la posición epistémica del prejuicio, al menos algunos de ellos, la que tiene ciertas implicancias que Spinoza quiere combatir.

Las consideraciones anteriores parece muy relevantes para comprender el genuino objetivo del TTP, que nada parece tener que ver con el establecimiento de un "mínimo doctrinal" o "mínimo teológico". Lo anterior se puede apreciar a partir

\footnotetext{
${ }^{5}$ Se puede ver también JAMES, 2012. Para un análisis más detenido del papel de estos conceptos en la "Introducción" del TTP se puede ver Placencia (2015).
} 
de los siguientes pasajes de la introducción a la obra, en la que Spinoza se refiere a la temática de la misma:

7) Ahora bien, el máximo secreto del régimen monárquico y su mayor interés consiste en engañar a los hombres y en enmascarar bajo el especioso nombre de 'religión' el miedo con que se los quiere controlar de modo que luchen por su esclavitud como si fuera su salvación (salus), y no consideren vergonzoso, sino que como el más grande honor, el dar su sangre y su vida por la vanidad de un solo hombre. En una comunidad política (republica) libre no se podría imaginar ni intentar nada más poco afortunado (infoelicius), puesto que es contrario a la libertad de cada uno el apropiarse del juicio libre de cada uno mediante prejuicios o coaccionarlo de algún modo. Y en lo concerniente a la sediciones, suscitadas bajo la apariencia de la religión, surgen sólo del hecho de que se dictan leyes sobre asuntos especulativos y que las opiniones son consideradas y condenadas como un delito, tal como si fueran crímenes. Los defensores y partisanos de estas no son inmolados por la salvación (publica salus), sino sólo por el odio y la crueldad de sus adversarios. No cabría ornar como derecho las sediciones antedichas, ni las controversias se transformarían en sediciones, si por la ley del Estado sólo se persiguieran los hechos, y los dichos permanecieran impunes.

8) Dado que nos toca en suerte la rara dicha de vivir en una comunidad política, donde le es concedido a cada uno una libertad íntegra de juicio y de rendir culto a Dios según su propio carácter (ingenium) y donde nada es más querido ni se tiene por más dulce que la libertad, creí <en consecuencia> que no hacía algo que fuera ingrato ni inútil si mostraba que esta libertad no sólo podía ser concedida preservando la piedad y la paz de la comunidad política, sino que además ella no puede ser eliminada sino junto con la misma paz de la comunidad política y la piedad. Y esto es lo que principalmente me propuse demostrar en este tratado. Para ello fue necesario, en primer lugar, indicar los prejuicios fundamentales sobre la religión, es decir, las huellas de la antigua esclavitud. Luego también fue necesario indicar los prejuicios sobre el derecho del soberano. Porque son muchos los que con desvergonzado desenfreno intentan arrebatarle una gran parte <de este derecho>, y bajo la apariencia de religión alejar del soberano el ánimo (animus) de la multitud, esclavo a tal punto de la superstición, de modo tal que todo caiga nuevamente en la esclavitud. Mencionaré aquí brevemente el orden en que mostraré esto, pero daré a conocer en primer lugar las causas que me han llevado a escribir (TTP Prefacio; G III 7).

Este texto, en el que parecen presentarse elementos importantes relativos al objetivo del libro evidentemente incluye elementos distintos a otro muy conocido como los centrales del texto, pero con ciertas coincidencias relevantes. Otro texto que confirma lo anterior es la Carta 30, en la que Spinoza se refiere por primera vez al TTP:

Ya compongo un tratado sobre mi opinión acerca de la Escritura. Me mueven a hacerlo las siguientes razones: i) Los prejuicios (praejudicia) de los teólogos. Sé, en efecto, que ellos son los que más impiden que los seres humanos puedan aplicar su ánimo a la filosofía. Por eso me esfuerzo en hacer manifiestos esos prejuicios y en apartarlos de las mentes de los más inteligentes; ii) La opinión que tiene de mí el vulgo, que no cesa de acusarme falsamente de ateísmo. Me siento constreñido a eliminarla cuanto sea posible; iii) La libertad de filosofar y decir lo que opinamos, que quiero asegurar de todos los modos. Aquí se la suprime completamente por la excesiva autoridad y petulancia de los predicadores. (Carta 30, Spinoza a H. Oldenburg 1665 [fragmento II]; G IV 166). 
De acuerdo a ambos textos, la tesis central del libro sería que la libertad de íntegra de juicio (judicandi libertas) y de rendir culto a Dios según su propio carácter es condición necesaria de la paz de la comunidad política y también de la piedad. Si esto es así, el libro es fundamentalmente un texto que muestra la necesidad de la libertad de expresión para la paz de la comunidad política (republica). Ello no parece hacerse apelando a ningún mínimo doctrinal, sino que parece apelar a la necesidad de luchar contra el prejuicio, i.e. el conjunto de dispositivos discursivos que buscan aprovechar la inestabilidad afectiva de los seres humanos. Se trata, como bien ja destacado Schnpef, no de eliminar tales prejuicios, sino que de indicarlos, para lograr, a su vez, no superar la superstición, sino que hacerla infectiva como insumo de manipulación política. Así las cosas, los prejuicios que se trata de combatir son "indicados", mas no "removidos", como ocurre en la Ética, según se lee en el Apéndice:

\begin{abstract}
Con esto, he explicado la naturaleza y las propiedades de Dios. Ellas son: 1) que existe necesariamente, 2) que es único, 3) que es y obra a partir de la sola necesidad de su naturaleza, 4) que es la causa libre de todo, 5) que todas las cosas son en Dios y que dependen de él mismo de modo que sin él no podrían ser ni ser concebidas, y 6) finalmente que todas las cosas han sido predeterminadas por Dios, ciertamente no por libertad de la voluntad, sino por la sola naturaleza y potencia infinita de Dios. Además, en cada lugar en que tuve la ocasión procuré quitar los prejuicios (praejudicia) que hubiesen podido impedir que mis demostraciones fueran comprendidas. Pero, dado que no restan pocos prejuicios que también hubieran podido y pueden impedir que los seres humanos comprendan la concatenación de las cosas por medio de aquello con lo que expliqué tal concatenación, he creído que valía la pena someterlas examen de la razón. Y dado que todos los prejuicios que aquí pretendo tomar dependen de uno sólo, sc. que los hombres suponen que todas las cosas naturales obran, tal como ellos, a causa de un fin (propter finem). Incluso, toman por hecho que el mismísimo Dios dirige todas las cosas a un cierto fin. Dicen, en efecto, que Dios hizo todas las cosas a causa de un fin (propter finem) y que hizo al hombre para que lo adore. Consideraré, ante todo, esto, buscando en primer lugar la causa por la que la mayor parte de los hombres descansan en este prejuicio y todos son por naturaleza a tal punto propensos abrazarlo. Luego, mostraré la falsedad de este prejuicio, y finalmente de qué modo han nacido de él aquellos del bien y el mal, el mérito y el pecado, la alabanza y la censura, el orden y la confusión, la belleza y la deformidad, y otros de ese género. (Ética l, apéndice)
\end{abstract}

\title{
Conclusión
}

Así las cosas, la búsqueda de Spinoza no consiste en evitar el conflicto incluso en el ámbito de las cuestiones religiosas, sino que más bien se trata de presentarlo, justamente, como una situación basada en afectos que pueden ser potencialmente empleados como instrumentos de manipulación política. Lo anterior permite al lector 
del texto reinterpretar sus prejuicios en la medida en que adquiere conciencia del carácter de tal de muchas de sus creencias o al menos adquiere elementos suficientes para sospechar la posibilidad de su carácter prejuicioso. Se trata de una perspectiva que evidentemente corresponde a una suerte de second best, puesto que en realidad lo óptimo sería algo que aparentemente es imposible, sc. remover los prejuicios, de modo que todos pudieran vivir filosóficamente. Frente a eso lo que cabe, nos enseña el TTP es, si no la "tolerancia", no el sentido original del término como "virtud de soportar", sino más bien entendida ella como una suerte de virtud autodistanciamiento de los propios juicios infundados, a lo menos una cierta "cautela preventiva”. En cualquiera de los casos, en ningún caso el objetivo del texto es una defensa de una concepción de la tolerancia como la que Svensson identifica con Spinoza, ni tampoco es sostenida esta interpretación en ningún lugar del texto. La posición de Spinoza parece vincularse más bien, como lo indica Mignini, con una idea de la tolerancia que no la considera como una virtud y que apela a que ella es una actitud que debería ser superada por quien ve las cosas filosóficamente. Es decir, justamente lo contrario del concepto que Svensson cree ver realizado en Spinoza. En el caso de quien no lleva una vida filosófica podría la tolerancia tener algún papel, pero en ningún caso bajo la especie del sometimiento a una mínimo doctrinal que elimine el conflicto, sino más bien desde una óptica similar, al menos, al enfoque Svensson identifica con la "mera tolerancia", i.e. desde la perspectiva de quien sosteniendo una visión sustantiva de lo que son el bien y el mal, admite y ni busca la supresión de la existencia de opiniones diferentes, pues ello entraña un mal aún mayor. En el caso que le interesa a Spinoza, i.e. la tentación a suprimir la libertad de expresión, el mal mayor sería la supresión de una comunidad política sana, que sería reemplazada por un régimen en el que se hacen del poder quienes con toda probabilidad buscan controlar a los miembros de esa comunidad por medio de dispositivos afectivos y de la generación de prejuicios.

\section{Referencias}

BELTRÁN, M. Toleranza e libertà di conscienza nell'opera di Spinoza: A proposto di una ipotesi di Filippo Mignini. Rivista di Filosofia Neo-Scolastica, v. 86, p. 738-746, 1994. 
JAMES, S. Spinoza on philosophy, religión and politics. Oxford: Oxford University Press, 2012.

MADANES, L. El árbitro arbitrario. Buenos Aires: EUDEBA, 2001.

MIGNINI, F. Nota di Filippo Mignini. Rivista di Filosofia Neo-Scolastica, v. 86, p. 747-749, 1994.

MIGNINI, F. ¿Más allá de la tolerancia? Trad. Silvio Mattoni, Córdoba: Editorial Brujas, 2009 (=1991).

PLACENCIA, L. Superstición, prejuicio y fortuna en Spinoza. Un breve comentario a la introducción del Tratado teológico-político. En: DE LOS RÍOS, I. (ed.). Azar: El sacro desorden de nuestras vidas. Madrid: Abada, 2015.

ROSENTHAL, M. Tolerance as a virtue in Spinoza's Ethics. Journal of the History of Philosophy, v. 39, p. 535-557, 2001.

SCHNEPF, R. Anlass und philosophische Grundlage des Theologisch-politischen Traktat sowie der Kontext in Spinozas Werk. En: HÖFFE, O. (ed.). Baruch Spinoza: Theologisch-politischer Traktat. Berlin: Akademie Verlag, 2014. p. 27-50.

SPINOZA, B. B. Spinoza. CEuvres III. Traité théologico-politique. Texte établi par Fokke Akkermann. Traduction et notes par Jacqueline Lagrée et Pierre-François Moreau. Paris: PUF, 1999. (Abreviado como TTP).

SPINOZA, B. Opera Omnia. Herausgegeben von C. Gebhardt. Heidelberg: C. Winter, 1925. (Abreviado como $G$ ).

SVENSSON, M. Hobbes, Spinoza y Locke sobre la herejía. Revista Pléyade, v. 8, p. 125-136, 2011.

SVENSSON, M. Una disposición pasajera. Santiago de Chile: Ediciones de la Universidad Diego Portales, 2013.

WALTHER, M. Der systematische Ertrag der exegetische Arbeit. En: HÖFFE, O. (ed.). Baruch Spinoza: Theologisch-politischer Traktat. Berlin: Akademie Verlag, 2014. p. 139-155.

RECIBIDO: 30/11/2019

APROBADO: $14 / 06 / 2020$

RECEIVED: $11 / 30 / 2019$

APPROVED: 06/14/2020

RECEBIDO: 30/11/2019

APROVADO: $14 / 06 / 2020$ 